grund gestellt.“ - „Wirtschaftliche Interessen stellen die Eigenschaften der Lanipe in anderem Lichte dar.“ - „Was ist danit er- reicht, wenn die Erythemstrahlung der , mittleren Sommersonne in Hamburg“ erreicht wird?" - „Wie sehen die Heilerfolge aus? In welcher Zeit und mit welchem Energieaufwand wurden sie erreicht?" -. „Die Lichtinstitute, die eine große Verantwortung übernehmen, dürfen an derartigen Fragen nicht vorübergehen."

Abgesehen von diesen Streitfragen verstehe ich nicht, wie man für die praktische Rachitisbehandlung oder für die Bestrahlung von Erwachsenen in der Praxis eine Lichtquelle empfehlen will, bei der jede Seite des Kindes eine halbe Stunde lang bèstrahlt werden soll, wo es doch andere Ultraviolettstrahler gibt, die die oft schwer aufrecht zu erhaltende Geduld der Kleinen nicht auf eine so harte Probe stellen. Soll bein Erwachsenen die Bestrahlung jeder Seite gar eine Stunde lang dauern? Wer hat die Zeit dazu?

Da die Lebensdauer einer Vitaluxlampe infolge ihrer hohen Ueberbelastung nur nit 300 Stunden und gegen Ende dieser Zeit für den noch strittigen, überhaupt vorhandenen geringen Ultraviolettgehalt ,ein Nachlassen von 20-25\%“ angegeben wird, vermag ich nicht einzusehen, daß diese Lampe ,wirtschaftlich" sein soll und nosh weniger, daß sie ,die Anwendung der Ultravioletttherapie in größerem Bereich als "bisher" erlaube. Für jeden kritischen Leser der Huldschinskyschen Arbeit, noch dazu unter Berücksichtigung der Kritiken von $\mathrm{R} \mathrm{ol}$ of $\mathrm{f}, \mathrm{K}$ e l le $\mathrm{r}$ und $\mathrm{K}$ ow a $\mathrm{rsch}$ ik ist die Vitaluxlampe als Ultraviolettlichtquelle nicht nur recht wenig brauchbar, sondern bedeutet auch Gefahr einer Diskreditierung der wahren Ultravioletttherapie mit echter Ultraviolettlichtquelle, wenigstens in all den Fällen, wo die Geduld des Patienten nicht die lange Zeit bis zum Erreichen einer Wirkung ausdauert. Bei den notwendigen, für die Praxis nahezu unmöglichen langen Bestrahlungszeiten wird der geringe Anschaffungspreis, der wegen der kurzen Lebensdauer der Lampe illusorisch ist, wettgemacht durch den vielen Stromverbrauch und die langen Bedienungsstunden. Die Vitaluxlampe kann lediglich gewisse günstige Wirkung haben, soweit eben Licht überhaupt günstig wirkt. Die Vitaluxlampe darf aber niemals zu den Ultraviolettlichtquellen gerechnet werden.

\title{
Ueber die Vitaluxlampe.
}

Von Geh. San.-Rat Hugo Bach, Weißer Hirsch-Dresden.

$\mathrm{Huldschinsky} \mathrm{empfiehlt} \mathrm{in} \mathrm{Nr.} 47$ die Vitaluxlampe als neue „ausreichende Ultraviolettlichtquelle, die eine Erythemwirkung erzeugt und rachitisverhütend wirkt", und wendet sich gegen die „Behauptungen einer bekannten Firma, daf die Vitaluxlampe nichts weiter darstelle als andere gewöhnliche Glühlampen". Ich nehnıe an, er meint mit der bekannten Firma die Quarzlampen-Gesellschaft, die aber, wie ich feststellen kann, gar nichts selbst behauptet, sondern sich auf die Feststellungen von $\mathrm{K}$ elle $\mathrm{r}$ und $\mathrm{K}$ ow a r s c hi k stützt. Beide Autoren haben die Vitaluxlampe eingehend geprüft, beiden gelang es nicht, ein Ultravioletterythem zu erzeugen, und beide lehnen es ab, die Vitaluxlampe zu den Ultraviolettlichtquellen zu rechnen. K ow a r s ch i k betont ausdrücklich, daß die therapeutische Wirkung der Vitaluxlampe im Wesentlichen eine $r$ ein the r in is $\mathrm{che}$ is und schreibt ferner wörtlich wie folgt: ,Zwar ist die Haut nach der Bestrablung intensiv gerötet. Diese Rötung ist aber bedingt durch eine Wärmehyperämie und nicht mit einem Lichterythein zu verwechseln. Denn erstens tritt sie schon unmittelbar während der Bestrahlung, nicht aber wie das Ultravioletterythem nach einer Latenzzeit von einigen Stunden auf. Zweitens ist die Hyperämie nicht eine rein kapillare, die Gefäßerweiterung betrifft vielmehr auch die Arteriolen. Die Rötung ist daher etwas fleckig, entsprechend dem Verlauf der kleinen Hautarterien. Dementsprechend fleckig ist auch die Pigmentierung, wie sie nach wiederholter Anwendung der Lampe bisweilen zustandekommt. Es ist die gleiche Pigmentierung, wie wir sie nach wiederholter Heißluftbehandlung beobachten. Die $t \mathrm{~h}$ e $\mathrm{r}$ apeutiseben Indikationen und i e therapeutischen Erfolge der Lampe entsprechendenender Solluxla nl pe, worüber neues kaum mehr zu sagen ist."

Huldschinsky. ist anscheinend der Verwechslung. eines bloßen Wärmeerythems mit dem charakteristischen Ultravioletterythem zum Opfer gefallen, denn auf S. 1963 bringt er eine Abbildung, welche "Ultravioletterytheme der Vitaluxlampe 2 Stunden 50 Minuten nach "beendeter Bestrahlung aufgenommen" zeigt. Ein wirkliches Ultravioletterythem ist, wie $\mathrm{Huldsch}$ in sky als alter Kenner der Hanauer Quarzlampe, die seinen Ruf begründet hat, doch wissen müßte, 3. Stunden nach der Bestrahlung noch gar nicht festzustellen, oder nur bei äußerst hoher Ultraviolettüberbestrahlung, die von vornherein $\mathrm{h}$ i e r ausgeschlossen ist. Sie tritt erst wesentlich später auf. Ferner übernimmt $\mathrm{H} \mathrm{u} \mathrm{ld} \mathrm{-}$ $\mathrm{sch}$ insky als physikalischen Beweis für das Vorhandensein einer Ultraviolettstrahlung bei der Vitaluxlampe ohne weiteres die Angaben der Herstellerfirma, die letzthin, ebenso wie die Leistungen und die Gutachten über die Vitaluxlampe eine vielbeachtete, geradezu vernichtende Kritik erfahren haben (R ol of f, Z. physik. Ther. 37 H. 4). Ich zitiere folgende drastische Schlagworte: "Probleme der Lichttherapie erfahren - absichtlich oder nicht - eine Behandlung, die zu schweren Bedenken Veranlassung gibt.“. „Physikalisehe Messungen, die außerdem rechnerisch korrigiert wurden, werden als Beweis für therapeutische Leistungen ausgelegt.“ "In der Propaganda wird Wesentliches unterdrückt und weniger Wichtiges in den Vorder- 